\title{
Prevalence of Cancer in Membranous Nephropathy: A Systematic Review and Meta-Analysis of Observational Studies
}

\author{
Napat Leeaphorn ${ }^{a}$ Pogsathorn Kue-A-Pai ${ }^{a}$ Natanong Thamcharoen ${ }^{a}$ \\ Patompong Ungprasert ${ }^{c}$ Michael B. Stokes ${ }^{b}$ Eric L. Knight ${ }^{a}$ \\ aDepartment of Internal Medicine, Bassett Medical Center and Columbia University College of Physicians and \\ Surgeons, Cooperstown, N.Y., and bepartment of Pathology, Columbia University College of Physicians and \\ Surgeons, New York, N.Y., USA; ' ${ }^{2}$ ivision of Rheumatology, Department of Medicine, Siriraj Hospital, Faculty of \\ Medicine, Mahidol University, Bangkok, Thailand
}

\section{Key Words}

Cancer · Epidemiology · Membranous nephropathy ·

Nephrotic syndrome $\cdot$ Phospholipase $A_{2}$ receptor

\begin{abstract}
Background: The association between membranous nephropathy (MN) and cancer has been well documented. However, the true prevalence and characteristics of cancer associated with MN have not been well described. Methods: A systematic review and meta-analysis of cohort studies was conducted to summarize the prevalence of cancer-associated MN as well as patient characteristics and types of cancer in this population. We used a random-effects meta-analysis model to estimate the prevalence of cancer. Results: We included 6 studies $(n=785)$. The estimated prevalence of cancer was $10.0 \%$ ( $95 \% \mathrm{Cl}, 6.1-14.6)$. The mean age of $\mathrm{MN}$ patients with cancer was $67 \pm 7$ years. The diagnosis of cancer preceded the diagnosis of MN in $20 \pm 6.8 \%$. Lung cancer was the most common type of tumor, accounting for 22 cases $(26 \%)$, followed by prostate cancer ( 13 cases, $15 \%)$, hematologic malignancies ( 12 cases, 14\%), colorectal cancer ( 9 cases, $11 \%)$, breast cancer (6 cases, $7 \%$ ), and stomach and
\end{abstract}

esophageal cancer (5 cases, 6\%). Conclusion: The estimated prevalence of cancer in patients with $\mathrm{MN}$ is $10 \%(95 \% \mathrm{Cl}, 6.1-$ 14.6). The vast majority of tumors associated with $\mathrm{MN}$ are lung and prostate cancer. Hematologic malignancies should also be considered as one of the potential cancers associated with MN. Our study was based on a largely Caucasian population; therefore, the findings might not be applicable to other populations.

(c) 2014 S. Karger AG, Basel

\section{Introduction}

Membranous nephropathy (MN) is a pathologic entity characterized by diffuse thickening of the glomerular basement membrane observed by light microscopy [1]. $\mathrm{MN}$ was historically considered the most common nephrotic syndrome in adults, but recent literature suggests that focal segmental glomerulosclerosis is now the leading cause of nephrotic syndrome [2]. Approximately 75\% of the cases of $\mathrm{MN}$ are idiopathic, whereas the remainder are associated with infections, malignancies, autoimmune diseases and drug toxicity [1]. 
The association between $\mathrm{MN}$ and cancer has been well recognized since 1966 when Lee et al. [3] reported that $11 \%$ of patients with nephrotic syndrome had carcinoma. However, the true prevalence of cancer in patients with $\mathrm{MN}$ remains unknown and has been variously estimated as ranging between 5 and 22\% [4-9]. Traditionally, the solid organ cancers most commonly associated with MN have been lung and gastrointestinal cancers [10], whereas $\mathrm{MN}$ associated with hematologic malignancy has been considered a rare entity [11].

The prevalence of cancer-associated $\mathrm{MN}$ varies across studies, in part due to variation in patient selection, follow-up periods, and different biopsy policies among centers. Furthermore, most studies to date have been limited in size given that $\mathrm{MN}$ is a relatively rare disease. Although several reviews have addressed the relationship between $\mathrm{MN}$ and the occurrence of cancer, to the best of our knowledge, no meta-analysis has been conducted to examine this relationship. We conducted a systematic review and meta-analysis to evaluate the prevalence of cancer in MN patients and the types of cancer associated with $\mathrm{MN}$ by reviewing existing observational study data.

\section{Materials and Methods}

\section{Data Sources and Searches}

The protocol for this review was developed according to standard reporting guidelines $[12,13]$. We conducted MEDLINE and Cochrane database searches of English-language studies undertaken in human subjects aged 18 or older using the following items: 'cancer AND glomerulopathy', 'cancer AND glomerular disease', 'cancer AND nephrotic syndrome', 'cancer AND membranous nephropathy', 'membranous nephropathy AND epidemiology'. The resulting publications identified with this search strategy as of February 7, 2014 were examined. We did not include articles based on hand searches of bibliographies, Internet searches or unpublished studies in the form of posters or abstracts in our search strategy.

\section{Study Selection and Data Extraction}

Studies pertaining to the determination of cancer risk in patients with $\mathrm{MN}$ were included for analyses if the following criteria were met: (1) they were cohort studies of patients with MN including prospective and/or retrospective studies and (2) data on cancer prevalence were demonstrated. Case reports, case series, cross-sectional studies, studies with uncertain follow-up adherence, studies that limited the population age, studies that did not specify the types of tumor, and studies that demonstrated incidence of cancer only after the time of diagnosis of $\mathrm{MN}$ (not prior to the diagnosis of MN) were excluded from our analyses. All identified abstracts were reviewed independently by 2 investigators (N.L. and P.U.). The quality of each study was independently evaluated by each investigator using the Newcastle-Ottawa quality assessment scale [14]. Discrepancies in data extraction and quality assessment were resolved through collateral discussion. For this study, we extracted the following variables: author's name(s), publication year, country where the study was conducted, period of follow-up, number of patients studied, number of cancers observed in the cohort, patients' age and gender, and the presence of cancer symptoms at the time of diagnosis of MN. We contacted the authors of the primary reports to request any unpublished data. If the authors did not reply, we used the available data for our analyses.

\section{Data Synthesis and Analysis}

The characteristics of MN patients with cancer enrolled in the study were reported as frequencies or mean \pm standard deviation. The prevalence of cancer and 95\% CIs were reported using a DerSimonian-Laird random-effects model with double arcsine transformation $[15,16]$. We ran a random-effect model rather than a fixed-effects model because of the high likelihood of heterogeneity between study variance. The heterogeneity of effect size estimates across studies was described with the $\mathrm{I}^{2}$ index and Q statistic's $\mathrm{p}$ value [17]. Analyses were performed using MetaXL software (http://www.epigear.com). The frequencies of each type of cancer were presented as a crude percentage.

\section{Results}

\section{Description of Included Studies}

The initial search yielded 2,482 articles (fig. 1). Of these, 2,379 were deemed ineligible because they were not related to the study question. We reviewed the full text of the remaining 35 articles for possible inclusion. A total of 13 studies were potentially eligible based on our inclusion criteria. Of these, 3 studies were excluded because of lack of follow-up data [18-20], 1 study did not report the total number of MN patients in the study [3], 1 study was based on $\mathrm{MN}$ patients aged 60 or over, so it did not represent the general MN population [7], 1 study did not specify the types of tumor that MN patients had [6], and 1 study described the incidence of cancers only after the diagnosis of $\mathrm{MN}$ was made [21]. Eventually, 6 cohort studies were included in the meta-analysis $[4-6,8,9,22]$. The characteristics of these studies are outlined in table 1. A total of 785 patients with MN were represented in these studies.

\section{Cancer Prevalence}

The prevalence of cancers within the 6 individual study populations ranged between 4.8 and $20.49 \%$ (crude unweighted mean $10.8 \%$ ), with an overall meta-analytical prevalence of $10.0 \%$ (95\% CI, 6.1-14.6), and there was evidence of a moderate level of heterogeneity $\left(\mathrm{I}^{2}=73.8 \%\right.$, $\mathrm{p}<0.001$; fig. 2).

\section{Reporting Bias}

A funnel plot was not drawn because of the limited number of studies. As a rule of thumb, tests for funnel
Leeaphorn/Kue-A-Pai/Thamcharoen/ Ungprasert/Stokes/Knight 
Fig. 1. Literature search flow diagram.

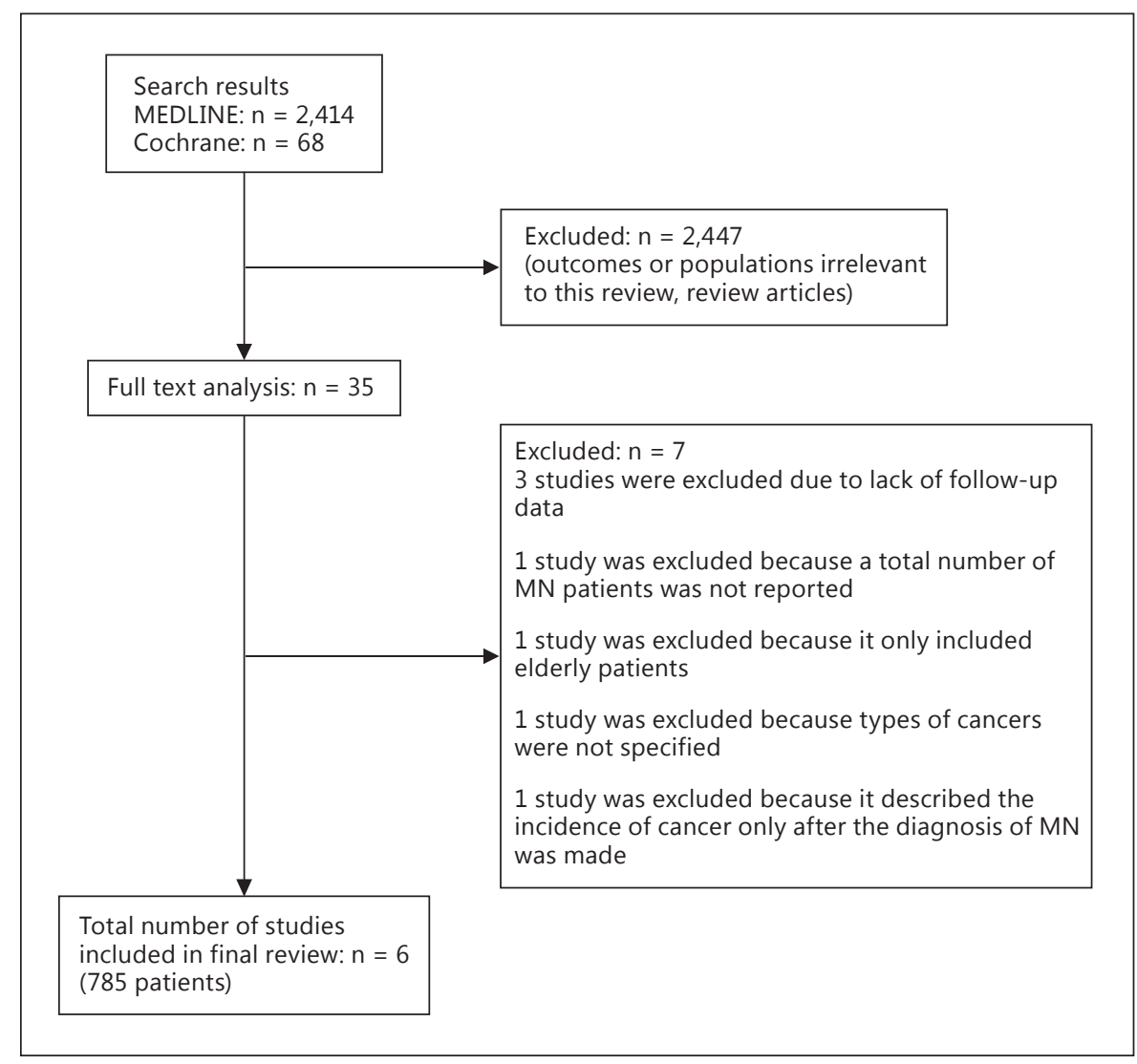

Table 1. Characteristics of cohort studies of cancer prevalence in patients with $\mathrm{MN}$ in the meta-analysis

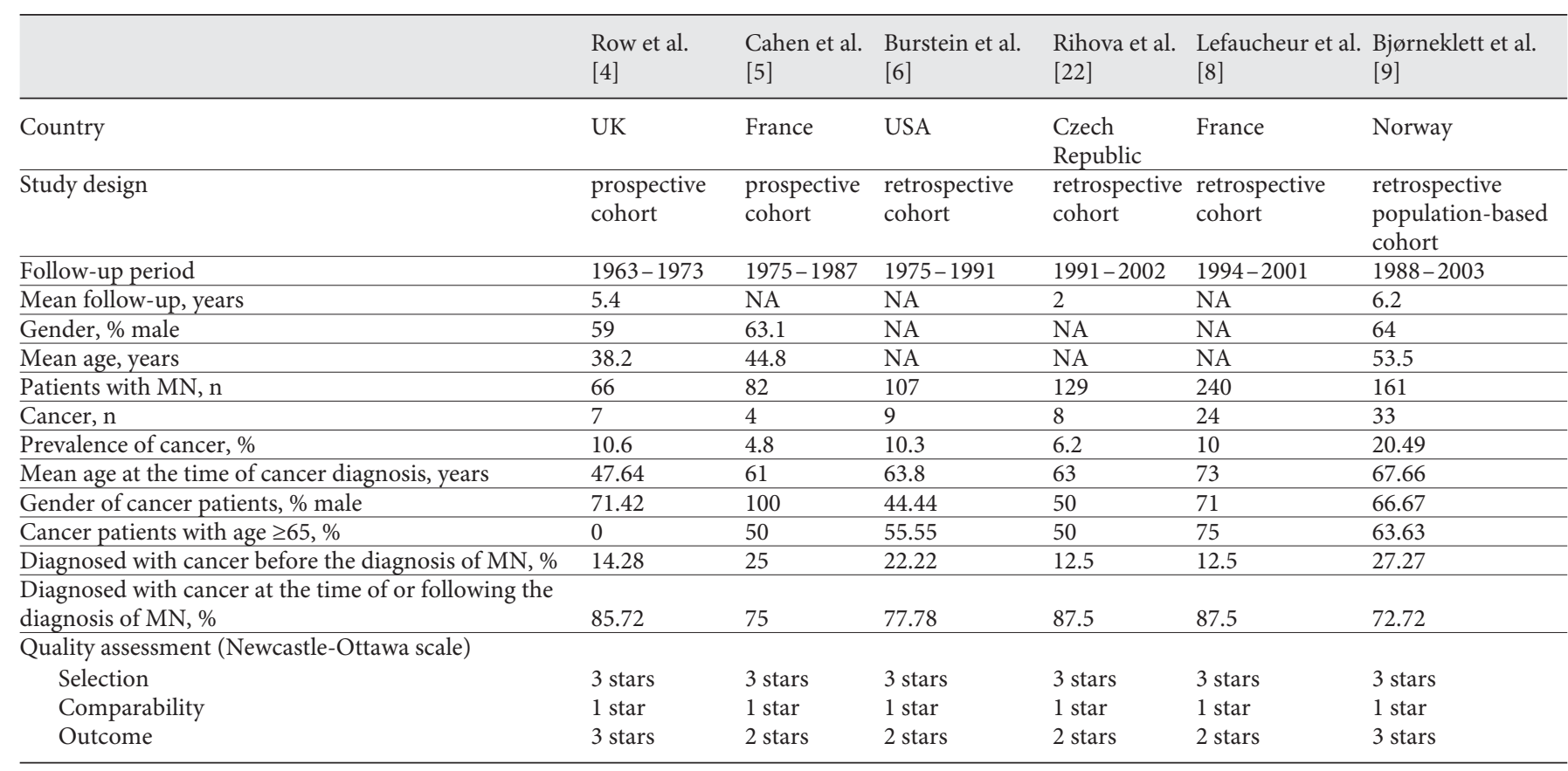

NA = Not available. 


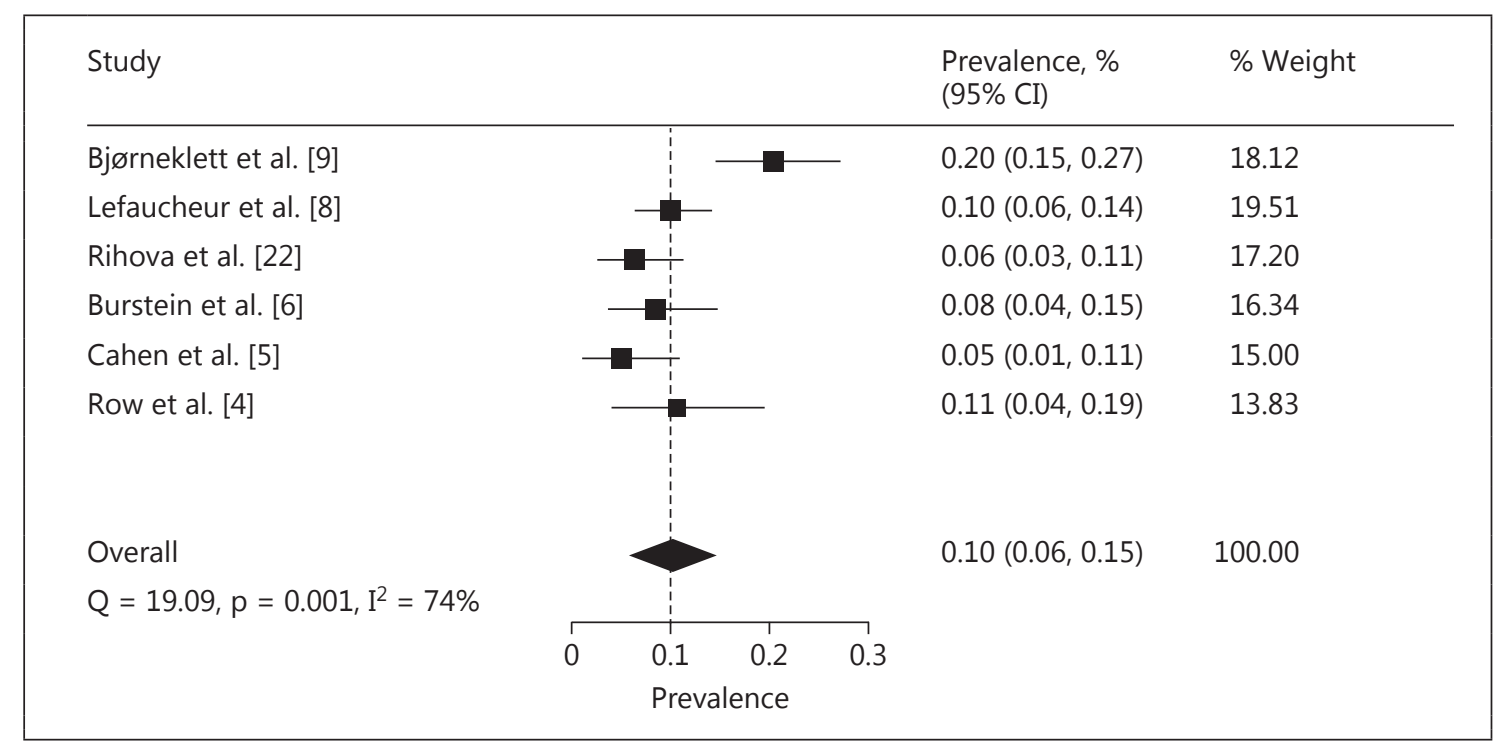

Fig. 2. Meta-analysis of cancer prevalence in MN populations.

plot asymmetry should be used only when there are at least ten study groups. With fewer studies, the power of the test is too low to distinguish chance from real asymmetry [23].

\section{Baseline Characteristics of Cancer-Associated MN}

Patients tended to present in their sixth to seventh decade with the mean age being $66 \pm 7$ years. Males represented up to $66 \%$ of all cancer patients with MN. Of all the cases of cancer associated with $\mathrm{MN}$ in all the studies combined, the cancer was discovered before the diagnosis of $\mathrm{MN}$ in only $20 \pm 6.8 \%$. For the remaining $80 \pm 15 \%$, the cancer was diagnosed at the time of or following the diagnosis of MN.

\section{Types of Cancer Associated with MN}

The vast majority of tumors associated with $\mathrm{MN}$ were solid tumors (73 cases, $86 \%$ ). Lung cancer was the most common type of solid tumor accounting for $26 \%$ (22 cases) followed by prostate cancer ( 13 cases, $15 \%$ ), colorectal cancer ( 9 cases, $11 \%)$, breast cancer ( 6 cases, $7 \%$ ), stomach and esophageal cancer ( 5 cases, $6 \%$ ), bladder cancer ( 4 cases, 5\%), cervical and uterine cancer (3 cases, $4 \%$ ) and renal cell carcinoma ( 2 cases, $2 \%$; table 2 ).

Out of 85 cases of cancer with MN, there were 12 cases $(14 \%)$ of hematologic malignancies. It could be noted that when all types of hematologic malignancies were combined, it became the third most common type of cancer after lung and prostate cancer. Lymphoma was the most common type of hematologic malignancy (6 cases; 3 cases of non-Hodgkin's lymphoma, 1 case of Hodgkin's lymphoma, and 2 cases that were unspecified), followed by chronic lymphocytic leukemia (4 cases), chronic myelogenous leukemia (1 case) and polycythemia vera (1 case).

\section{Discussion}

This study presents, to our knowledge, the first systematic review and meta-analysis of cancer prevalence in patients with MN. We confirmed a close association between $\mathrm{MN}$ and cancer with a prevalence rate of $10 \%(95 \%$ CI, 6.1-14.6). It should be noted that we did not take standardized incidence ratio into account for the analyses because there were only 2 cohort studies that reported this $[8,9]$. Standardized incidence ratio would have been a better statistical tool to compare the incidence rate of cancer in $\mathrm{MN}$ patients with that in the general population.

Heterogeneity between studies was present in this meta-analysis. We suspect that the difference in follow-up periods was the main source of heterogeneity, as half of the studies did not report the mean length of follow-up, and the mean follow-up also varied between 2 and 6.2 years in the remaining studies. Theoretically, the longer the follow-up period the higher the number of cancer cases that would be reported, as evident by the work of Bjørneklett et al. [9], who stated that the risk of develop-
Leeaphorn/Kue-A-Pai/Thamcharoen/ Ungprasert/Stokes/Knight 
Table 2. Overall and individual study prevalence of specific types of cancer

\begin{tabular}{|c|c|c|c|c|c|c|c|}
\hline Type of malignancy & $\begin{array}{l}\text { Bjørneklett } \\
\text { et al. [9] }\end{array}$ & $\begin{array}{l}\text { Lefaucheur } \\
\text { et al. }[8]\end{array}$ & $\begin{array}{l}\text { Rihova } \\
\text { et al. [22] }\end{array}$ & $\begin{array}{l}\text { Burstein } \\
\text { et al. [6] }\end{array}$ & $\begin{array}{l}\text { Cahen } \\
\text { et al. [5] }\end{array}$ & $\begin{array}{l}\text { Row } \\
\text { et al. [4] }\end{array}$ & Total \\
\hline Lung & $6(18.18)$ & $8(33.33)$ & $3(37.5)$ & $2(22.22)$ & $1(25)$ & $2(28.57)$ & $22(25.88)$ \\
\hline Hematologic & $5(15.15)$ & $2(8.33)$ & $0(0)$ & $2(22.22)$ & $1(25)$ & $2(28.57)$ & $12(14.11)$ \\
\hline Colon/rectal & $4(12.12)$ & $1(4.16)$ & $1(12.5)$ & $1(11.11)$ & $0(0)$ & $2(28.57)$ & $9(10.58)$ \\
\hline Breast & $4(12.12)$ & $1(4.16)$ & $0(0)$ & $1(11.11)$ & $0(0)$ & $0(0)$ & $6(7)$ \\
\hline Bladder & $2(6)$ & $1(4.16)$ & $0(0)$ & $1(11.11)$ & $0(0)$ & $0(0)$ & $4(4.7)$ \\
\hline Renal cell carcinoma & $0(0)$ & $0(0)$ & $0(0)$ & $1(11.11)$ & $1(25)$ & $0(0)$ & $2(2.35)$ \\
\hline Larynx & $0(0)$ & $1(4.16)$ & $0(0)$ & $0(0)$ & $0(0)$ & $0(0)$ & $1(1.17)$ \\
\hline Thymus & $0(0)$ & $1(4.16)$ & $0(0)$ & $0(0)$ & $0(0)$ & $0(0)$ & $1(1.17)$ \\
\hline Mediastinum & $0(0)$ & $1(4.16)$ & $0(0)$ & $0(0)$ & $0(0)$ & $0(0)$ & $1(1.17)$ \\
\hline Cervix/uterus & $1(3)$ & $1(4.16)$ & $1(12.5)$ & $0(0)$ & $0(0)$ & $0(0)$ & $3(3.5)$ \\
\hline Disseminated, unknown & $1(3)$ & $0(0)$ & $0(0)$ & $0(0)$ & $0(0)$ & $0(0)$ & $1(1.17)$ \\
\hline Wilms' tumor & $0(0)$ & $0(0)$ & $0(0)$ & $0(0)$ & $0(0)$ & $1(14.28)$ & $1(1.17)$ \\
\hline
\end{tabular}

Values are number of cases (percentage). Number and percent of cases (in parentheses).

ing cancer persisted for at least 5 years after the diagnosis of MN [9]. In addition, methodological differences in cancer detection might also play a role in the heterogeneity in our study. Bjørneklett et al. [9] identified cancer in patients with $\mathrm{MN}$ by using record linkage with national cancer registries, whereas other studies identified cancer by accessing patient charts from their own institutions. The biopsy practices and policies among centers might also cause variation in cancer prevalence as some nephrologists might be reluctant to perform a kidney biopsy in patients with symptomatic cancer as the biopsy results would not be likely to change the management.

Not surprisingly, the incidence of cancer associated with $\mathrm{MN}$ increases with age $[7,21]$. Lefaucheur et al. [8] reported that the frequency of cancer was up to $20-25 \%$ after age 60 years. In our analyses, the mean age of MN patients with cancer was $66.35 \pm 6.75$ years. Interestingly, only $20 \pm 6.8 \%$ of MN patients had the diagnosis of cancer before the diagnosis of MN. Cancer was discovered at the time of or following the diagnosis of $\mathrm{MN}$ in the remainder. This finding is important because it emphasizes the benefit of cancer screening in patients with newly diagnosed MN. There are, however, two shortcomings worth mentioning because of insufficient data. First, we were unable to further subdivide the proportions of patients whose cancer was discovered concomitantly with the di- agnosis of MN or during the follow-up period. Secondly, it would have been even more interesting clinically if it were possible to learn the percentage of patients who already had cancer-related symptoms at the time of renal biopsy. Lefaucheur et al. [8] reported that $52 \%$ of all patients with cancer-associated MN who did not have the diagnosis of cancer at the time of renal biopsy had cancerrelated symptoms such as cough, urinary retention, and impairment of general health status. Unfortunately, the remaining 5 studies did not mention cancer-related symptoms. None of the studies mentioned the rate of thromboembolic events. Lefaucheur et al. [8] and Bjørneklett et al. [9] demonstrated no association between the development of cancer and the degree of proteinuria, serum albumin or other laboratory parameters.

In our study, we did not have enough data to analyze the median time from diagnosis of $\mathrm{MN}$ to cancer in $\mathrm{MN}$ patients in whom cancer was discovered during the follow-up period. However, Bjørneklett et al. [9] reported that the median time was 5 years, which suggests that the predilection for cancer in participants with MN persists for many years. Therefore, close follow-up is necessary if cancer is not detected on initial screening.

It has been well documented in the literature that $\mathrm{MN}$ is closely associated with solid tumors rather than with hematologic malignancies. In our analyses, lung cancer 
was the most common solid tumor, accounting for almost a quarter of all cancer patients, followed by prostate cancer, colorectal cancer, breast cancer and stomach and esophageal cancer, respectively. It is probably worth noting that the high frequency of prostate cancer was overwhelmingly reported from the 2 most recent studies [8, 9]. This finding may be explained by a better detection of this slow-growing tumor by prostate-specific antigen testing and modern imaging [24].

Interestingly, we found that the frequency of hematologic malignancies was also high, the third rank after lung and prostate cancer. Lymphoma (6 cases) was the most common hematologic malignancy associated with $\mathrm{MN}$ followed by chronic lymphocytic leukemia (4 cases). To date, $\mathrm{MN}$ associated with hematologic malignancies has only been described in isolated case reports $[11,25]$. Our findings suggest that the clinicians should be more vigilant searching for hematologic malignancies.

The precise pathogenic mechanism whereby cancer might be associated with MN has yet to be elucidated. Beck [26] proposed four potential mechanisms: (1) antibodies may be created against a tumor antigen immunologically similar to an endogenous podocyte antigen, thereby leading to in situ immune complex formation; (2) shed tumor antigens may form circulating immune complexes that are subsequently trapped in the glomerular capillary wall; (3) circulating antibodies may also react to the tumor antigens that have already been planted in the subepithelial location, or (4) an extrinsic process such as viral infection or an underlying abnormal immune response may be responsible for both cancer and $\mathrm{MN}$.

Since the discovery of the transmembrane glycoprotein M-type phospholipase $A_{2}$ receptor $\left(P_{L A} R\right)$, which was identified as the major target podocyte antigen involved in the majority of adult idiopathic MN cases [24], a distinction between idiopathic and secondary $\mathrm{MN}$ can be made more precisely by the detection of circulating antibodies against $\mathrm{PLA}_{2} \mathrm{R}$. The patients with $\mathrm{MN}$ who have detectable anti-PLA ${ }_{2} \mathrm{R}$ are unlikely to have associated malignancy, whereas the absence of anti-PLA ${ }_{2} \mathrm{R}$ increases the risk of malignancy-associated $\mathrm{MN}$ and warrants careful searches for cancer [27]. Note that an anti$\mathrm{PLA}_{2} \mathrm{R}$ autoantibody assay has not been approved by the US Food and Drug Administration at the time of this writing, although it is available in Europe [28]. Glomerular pathologic findings are also very useful. Strong immunofluorescence staining of glomerular $\mathrm{PLA}_{2} \mathrm{R} \operatorname{IgG1}$ and IgG2 is frequently seen with cancer-associated $\mathrm{MN}$, whereas the dominance of deposits of IgG4 generally fa- vors idiopathic MN $[29,30]$. However, Larsen et al. [31] did find positive staining with IgG4 predominance in 3 out of 12 patients (25\%) who had malignancy-associated MN. The question remains whether these patients had coincidental/unrelated malignancies that were detected after the diagnosis of primary MN. In addition to the above, the presence of more than eight inflammatory cells infiltrating the glomeruli seems to strongly increase the likelihood of cancer in patients with $\mathrm{MN}$ [8].

It has now become standard practice to search for malignancy in older patients with newly diagnosed $\mathrm{MN}$ once other secondary causes have been excluded. However, there is no consensus on how aggressive clinicians should be in search of occult malignancy. Recent literature based on expert opinion suggests vigorous pursuit for cancer if circulating anti-PLA $\mathrm{A}_{2} \mathrm{R}$ autoantibodies are undetected and/or IgG deposits are not IgG4 subclass-dominant and/ or there are more than eight inflammatory cells infiltrating the glomeruli $[1,32,33]$. Based on our results showing a relatively high prevalence of cancer in the MN population, especially in older patients, we concur with this approach. If cancer is not detected on initial screening, these patients should have close medical follow-up because of the long-term risk of cancer occurrence.

Our analysis should be interpreted with caution. First, the observational studies were moderately heterogeneous and thus publication bias and a residual confounding bias may have existed although we cannot assess these hypotheses. Secondly, overall numbers of patients in each study and overall were relatively small, and long-term follow-up was variable. Thirdly, all of the studies included were conducted in developed Western countries with the majority of the subjects being Caucasian, therefore our findings may not represent $\mathrm{MN}$ populations from other parts of the world. Lastly, we could not judge whether the occurrence of cancer in this study was a causative factor for developing MN or a mere coincidence. Given that MN populations were likely to be elderly patients who were already at high risk for developing cancer, it is possible that some of the cancer cases in the study were just coincidental.

In conclusion, the results of our meta-analysis confirm a high prevalence of cancer in the MN population. The malignancies most frequently associated with $\mathrm{MN}$ are lung and prostate cancer, followed by colorectal cancer, breast cancer and lymphoma. Further study is warranted to determine the true incidence of cancer in $\mathrm{MN}$ patients with negative anti-PLA ${ }_{2} \mathrm{R} 1$ autoantibodies and/or the absence of IgG4 subclass dominance by immunofluorescence and/or the presence of more than eight inflamma-
Leeaphorn/Kue-A-Pai/Thamcharoen/ Ungprasert/Stokes/Knight 
tory cells. Until such information is available, it is reasonable to pursue aggressive cancer screening in patients with these serologic and glomerular morphologic pictures. Systemic investigation for possible lung and prostate cancer should be the first priority. Hematologic malignancies should also be considered as a potential cause of $\mathrm{MN}$.

\section{Disclosure Statement}

The authors declare that they have no financial conflict of interest related to this study.

\section{References}

1 Ponticelli C, Glassock RJ: Glomerular diseases: membranous nephropathy - a modern view. Clin J Am Soc Nephrol 2014;9:609-616.

$>2$ Swaminathan S, Leung N, Lager DJ, Melton LJ 3rd, Bergstralh EJ, Rohlinger A, Fervenza FC: Changing incidence of glomerular disease in Olmsted County, Minnesota: a 30-year renal biopsy study. Clin J Am Soc Nephrol 2006; 1:483-487.

$\checkmark 3$ Lee JC, Yamauchi H, Hopper J Jr: The association of cancer and the nephrotic syndrome. Ann Intern Med 1966;64:41-51.

$\checkmark 4$ Row PG, Cameron JS, Turner DR, Evans DJ, White RH, Ogg CS, Chantler C, Brown CB: Membranous nephropathy. Long-term follow-up and association with neoplasia. Q J Med 1975;44:207-239.

5 Cahen R, Francois B, Trolliet P, Gilly J, Parchoux B: Aetiology of membranous glomerulonephritis: a prospective study of 82 adult patients. Nephrol Dial Transplant 1989;4: 172-180.

6 Burstein DM, Korbet SM, Schwartz MM: Membranous glomerulonephritis and malignancy. Am J Kidney Dis 1993;22:5-10.

$>7$ Zech P, Colon S, Pointet P, Deteix P, Labeeuw $\mathrm{M}$, Leitienne P: The nephrotic syndrome in adults aged over 60: etiology, evolution and treatment of 76 cases. Clin Nephrol 1982;17: 232-236.

8 Lefaucheur C, Stengel B, Nochy D, Martel P, Hill GS, Jacquot C, Rossert J, GN-PROGRESS Study Group: Membranous nephropathy and cancer: epidemiologic evidence and determinants of high-risk cancer association. Kidney Int 2006;70:1510-1517.

-9 Bjørneklett R, Vikse BE, Svarstad E, Aasarød K, Bostad L, Langmark F, Iversen BM: Longterm risk of cancer in membranous nephropathy patients. Am J Kidney Dis 2007;50:396403.

$>10$ Bacchetta J, Juillard L, Cochat P, Droz JP: Paraneoplastic glomerular diseases and malignancies. Crit Rev Oncol Hematol 2009; 70 : 39-58.

$>11$ Mallouk A, Pham PT, Pham PC: Concurrent FSGS and Hodgkin's lymphoma: case report and literature review on the link between nephrotic glomerulopathies and hematological malignancies. Clin Exp Nephrol 2006;10: 284-289.
12 STROBE statement - checklist of items that should be included in reports of observational studies (STROBE initiative). Int J Public Health 2008;53:3-4.

13 Stroup DF, Berlin JA, Morton SC, Olkin I, Williamson GD, Rennie D, Moher D, Becker BJ, Sipe TA, Thacker SB: Meta-analysis of observational studies in epidemiology: a proposal for reporting. Meta-analysis Of Observational Studies in Epidemiology (MOOSE) group. JAMA 2000;283:2008-2012.

14 Stang A: Critical evaluation of the NewcastleOttawa scale for the assessment of the quality of nonrandomized studies in meta-analyses. Eur J Epidemiol 2010;25:603-605.

15 DerSimonian R, Kacker R: Random-effects model for meta-analysis of clinical trials: an update. Contemp Clin Trials 2007;28:105114.

16 Barendregt JJ, Doi SA, Lee YY, Norman RE, Vos T: Meta-analysis of prevalence. J Epidemiol Community Health 2013;67:974-978.

17 Higgins JP, Thompson SG, Deeks JJ, Altman DG: Measuring inconsistency in meta-analyses. BMJ 2003;327:557-560.

18 Brueggemeyer CD, Ramirez G: Membranous nephropathy: a concern for malignancy. Am J Kidney Dis 1987;9:23-26.

19 Zeng CH, Chen HM, Wang RS, Chen Y, Zhang SH, Liu L, Li LS, Liu ZH: Etiology and clinical characteristics of membranous nephropathy in Chinese patients. Am J Kidney Dis 2008;52:691-698.

20 Yokoyama H, Taguchi T, Sugiyama H, Sato $\mathrm{H}$, Committee for the Standardization of Renal Pathological Diagnosis and for Renal Biopsy and Disease Registry in the Japanese Society of Nephrology: Membranous nephropathy in Japan: analysis of the Japan Renal Biopsy Registry (J-RBR). Clin Exp Nephrol 2012;16:557-563.

21 O'Callaghan CA, Hicks J, Doll H, Sacks SH, Cameron JS: Characteristics and outcome of membranous nephropathy in older patients. Int Urol Nephrol 2002;33:157-165.

22 Rihova Z, Honsova E, Merta M, Jancova E, Rysava R, Reiterova J, Zabka J, Tesar V: Secondary membranous nephropathy - one center experience. Ren Fail 2005;27:397-402.
23 Higgins J GS: Cochrane Handbook for Systematic Reviews of Interventions, version $5 \cdot 0 \cdot 1$ (updated March 2011). The Cochrane Collaboration, 2011.

24 Beck LH Jr, Bonegio RG, Lambeau G, Beck DM, Powell DW, Cummins TD, Klein JB, Salant DJ: M-type phospholipase A2 receptor as target antigen in idiopathic membranous nephropathy. N Engl J Med 2009;361:11-21.

25 Ronco PM: Paraneoplastic glomerulopathies: new insights into an old entity. Kidney Int 1999;56:355-377.

26 Beck LH Jr: Membranous nephropathy and malignancy. Semin Nephrol 2010;30:635644.

27 Timmermans SA, Ayalon R, van Paassen P, Beck LH Jr, van Rie H, Wirtz JJ, Verseput GH, Frenken LA, Salant DJ, Cohen Tervaert JW, Limburg Renal R: Anti-phospholipase A2 receptor antibodies and malignancy in membranous nephropathy. Am J Kidney Dis 2013; 62:1223-1225.

28 Jhaveri KD, Shah HH, Patel C, Kadiyala A, Stokes MB, Radhakrishnan J: Glomerular diseases associated with cancer, chemotherapy, and hematopoietic stem cell transplantation. Adv Chronic Kidney Dis 2014;21:48-55.

-29 Qu Z, Liu G, Li J, Wu LH, Tan Y, Zheng X, Ao J, Zhao MH: Absence of glomerular IgG4 deposition in patients with membranous nephropathy may indicate malignancy. Nephrol Dial Transplant 2012;27:1931-1937.

30 Ohtani H, Wakui H, Komatsuda A, Okuyama S, Masai R, Maki N, Kigawa A, Sawada K, Imai $\mathrm{H}$ : Distribution of glomerular IgG subclass deposits in malignancy-associated membranous nephropathy. Nephrol Dial Transplant 2004; 19:574-579.

-31 Larsen CP, Messias NC, Silva FG, Messias E, Walker PD: Determination of primary versus secondary membranous glomerulopathy utilizing phospholipase A2 receptor staining in renal biopsies. Mod Pathol 2013;26:709-715.

32 Cambier JF, Ronco P: Onco-nephrology: glomerular diseases with cancer. Clin J Am Soc Nephrol 2012;7:1701-1712.

33 Glassock RJ: Attending rounds: an older patient with nephrotic syndrome. Clin J Am Soc Nephrol 2012;7:665-670. 